\title{
Study on Construction and Improvement of Community Public Sports Service Evaluation System under View of Nationwide Fitness
}

\author{
Jun Hu, Hefang Liu \\ Institute of Physical Education, Baoshan University, Baoshan Yunnan, 678000, China
}

Keywords: Nationwide fitness, Community public sports, Service evaluation system

\begin{abstract}
To further improve public service level of Chinese urban community and offer corresponding guarantee for intensifying physical quality of urban residents, this paper is based on social background of nationwide fitness and plans to adopt fuzzy analytical hierarchy process (AHP) to further analyze construction and improvement of community public sports service evaluation system, and adopts the improvement measures according to the analysis results, in the hope of offering corresponding theoretical support for construction and development of community public sports service evaluation system, gradually driving nationwide fitness and boosting overall quality of the public.
\end{abstract}

\section{Introduction}

In the new period, as socialist market economy develops and people's living level improves, the public gain material life guarantee. On this basis, the public turn the attention to comprehensive quality and good health. Hence, physical exercise receives extensive attention of the public, which generates positive influence on improvement of nationwide fitness. Nowadays, nationwide fitness idea has enjoyed popular support, which drives sound development of physical education. Thus, based on social background of nationwide fitness, it is required to strengthen community sports public sports service construction in urban construction process, construct scientific public service evaluation system, ensure service quality of community public sports and contribute to nationwide fitness activity in order to meet public demand for physical exercise.

\section{Relationship between Nationwide fitness Idea and Community Public Sports Service}

Community is the foundation for further construction and development of cities and society, and also an important carrier of implementing nationwide fitness policy, which offers the platform for urban residents to participate in physical exercise and generates certain influence on the development of urban sports cause. Community public sports service is an important constituent part of community sports construction. Its development objective is to provide convenient and fast sports products and public services for community residents. It is beneficial to drive implementation of citywide fitness program $^{[1]}$. Hence, under social background of nationwide fitness, it is required to actively implement correct thought of nationwide fitness during carrying out public service of community public sports, satisfy sports development requirement of urban community residents and offer corresponding guarantee for implementing nationwide fitness under the leadership of government sector and active participation of all sectors of society. Meanwhile, the State Council also clearly points out in the guiding opinion on sports work in the new period that, offices in villages and towns and government sector should pay more attention to nationwide fitness, incorporate nationwide fitness idea in community public service system, intensify construction and improvement of community public sports service evaluation system, drive development of urban sports construction and facilitate implementation of nationwide fitness program. 


\section{Study on Construction and Improvement of Community Public Sports Service Evaluation System under View of Nationwide Fitness}

\section{Construction of Community Public Sports Service Evaluation System Based on Nationwide Fitness Background}

In current stage, as nationwide fitness idea penetrates in society and nationwide fitness idea influences sports awareness of the public, urban community public sports service in China has gained certain development, and sports service evaluation system construction also obtained periodical achievements. But some problems still exist and impose adverse effects on evaluation work and evaluation system construction. Hence, social sports sector should attach importance to these problems, adopt some methods to construct more sound and perfect service evaluation system and promote better development of community public service.

In recent years, under social background where socialist market economy keeps improving, China's urbanization course gradually speeds up, and urban population increases sharply, which to some extent gives rise to tight urban land, results in the increase in community public sports service cost and challenges construction of community public sports service system. Tight urban land will inevitably lead to the decrease in sports field used to carry out public sports service by the community. Both quantity and quality of sports activity are adversely affected, and residents' enthusiasm for participation in sports is greatly restrained. This is adverse to sustainable and sound development of urban community public sports service evaluation ${ }^{[2]}$. In addition, since China lacks attention to implementation of nationwide sports work in early construction, representative folk sports education and guidance organization is not formed yet. Thus, certain fusion obstacle exists between implementation of nationwide sports policy and public participation in sports, which is to the disadvantage of generalizing urban community public sports service system and imposes adverse impacts on evaluation system construction.

Meanwhile, complex influence factors in implementation of urban community public sports service under the view of nationwide fitness. To guarantee authenticity and objectivity of evaluation work, relevant workers may combine actual conditions and adopt fuzzy AHP to analyze and evaluate community public sports service system so as to get relatively reliable and objective data results. Simply speaking, fuzzy AHP organically combines fuzzy research theory and advanced analysis method - AHP. The general application process is divided into four links: 1) explore internal relations among evolution criteria of each factor in the evacuation system, and combine the relations to establish corresponding ladder-type progressive AHP model; 2) for the evolution indexes of the same hierarchy in AHP model, compare and study each other according to specific principles and then establish scientific fuzzy analytical judgment matrix; 3) check all evaluation factors and their relations, adjust fuzzy matrix which cannot reach consistency with numerous factors until consistency is achieved and then carry out next research; 4) calculate the weight of urban community sports service system under different hierarchy of evaluation principles in accordance with the weight of lower-layer evaluation indexes relative to upper-layer indexes. To apply this method to confirm weight coefficient of subjective evaluation indexes, firstly, basic structure model of fuzzy AHP should be confirmed. In other words, based on current community public sports service evaluation system, the demands of community public sports construction are combined to construct service evaluation system which conform to basic development situation of community public sports service and include evaluation index, principle and basis, and this relatively scientific service evaluation system is combined to improve structure model of fuzzy AHP. Secondly, corresponding fuzzy judgment matrix needs to be constructed according to evaluation needs, and experts should be invited to demonstrate the matrix and reflect relations among evaluation indexes so as to objectively and reliably evaluate community public sports service ${ }^{[3]}$.

Moreover, since community public sports service is influenced by numerous factors under the influence of current nationwide fitness environment, actual conditions should be combined to solve the problems in current community public sports service system in the process of constructing and improving scientific evaluation system and gradually improve community public sports service in 
order to ensure scientificity of evaluation system. In terms of specific operation, relevant community public sports service sector should stick to government's leadership, call upon the masses to take active part in it, promote harmonious exchange between the government and the masses, form benign interaction and boost scientificity of community public sports service system. Meanwhile, to boost deep development of nationwide fitness, the government should increase economic input in construction of community public sports service system, strengthen infrastructure construction and lay solid material basis for constructing service system ${ }^{[4]}$. Additionally, cultivation of high-quality professional talents is also the key content of community public sports service system construction. Relevant sectors should fully excavate the potential of high-quality professional talents, transfer professional physical exercise knowledge to the public, and facilitate better development of community public sports service system construction. Only when these problems are solved can relatively scientific evaluation system be established through all-round analysis. Meanwhile, the foundation can be laid for deep implementation of community public sports service evaluation under nationwide fitness background.

\section{Construction and Improvement of Community Public Sports Service Evaluation System Based on Nationwide Fitness Background}

The improvement of community public sports service evaluation system under the view of nationwide fitness involves multiple aspects of factors such as content, principle and index. Only scientific analysis and research can guarantee objectivity and reliability of evaluation system and contribute to implementation of community public sports service evaluation. This paper proceeds from service subject, object, target and environment and hopes to make corresponding contributions to constructing and improving evaluation system.

Firstly, for the subject of community public sports service work, it is known from fuzzy AHP research that, community public sports service management occupies an important position and mainly plays a coordination role in popularizing and promoting, community public sports service. Even the mentality, knowledge structure and professional ethics of community public sports management personnel generate decisive effects on service quality and restrict implementation of nationwide fitness activity. Hence, the construction of evaluation system should take the subject of community public sports as the key investigation object to ensure comprehensiveness of evaluation system.

Secondly, for the object of community public sports service work, the object of public sports service directly presents community public sports service quality. The public sports, interestingness and scientificity of sports activity are the key factors which influence the public to take active part in sports activity. In addition, the infrastructure also exerts corresponding influence on service evaluation. Thus, relevant management sector should enhance attention to infrastructure construction, actively introduce advanced information technology and abundant sport resources to promote prosperity and development of community public sports service and create conditions for constructing and improving service evaluation system.

Thirdly, for basic service goal of community public sports, the achievement of basic development goal of community public sports service has important effects on public sports service evaluation under the view of nationwide fitness. The selection and setting of concrete and quantitative service goal create conditions for service execution. Moreover, scientific and reasonable development goal may be set in service system construction process. Through all-round control of goal execution process, the gap between the achieved goal and the goal which is not achieved can narrow. Then, the reason for the gap should be explored, and countermeasures should be formulated according to the reason. In this way, more scientific and standard community public sports service system can be constructed to lay a foundation for improvement of evaluation system ${ }^{[5]}$.

Finally, for the environment of community public sports service, it is the object corresponding to the subject of community public sports service work and also the sum of various factors correlating, restricting and influencing community public sports service. Generally, the environment can be divided into natural environment and social environment. Natural environment refers to physical 
environment which can make sports service operate and generate specific influence on sports activity, such as air quality, community green area and sanitary conditions. Social environment mainly refers to economic and cultural factors, and corresponding guarantee can be offered for community public sports service system construction through mutual promotion and restriction of economic and cultural environmental factors. Favorable natural environment can promote the public generate a sense of joy during participation in physical exercise, provide more high-quality sports activity space for community masses, improve sports quality and reduce exercise risk through certain green land. Economic things generate fundamental influence on social development. Continuous economic growth drives the improvement of living standards and also lays a solid foundation for implementation of public sports service under the view of nationwide fitness. In current stage, benign implementation of urban community nationwide fitness service is influenced by urban economic level. To facilitate sustainable and healthy development of urban community public sports, relevant government sector should offer corresponding financial support for service system construction, guide the public to consciously participate in community public sports activity, gradually expand the team of nationwide construction and create conditions for improvement of national quality. Therefore, public sports service environment must serve as a significant factor for construction and improvement of service evaluation system. It is required to boost public service evaluation quality and facilitate better development of community public sports service under the view of nationwide fitness through objective analysis of environmental problem.

\section{Conclusions}

In conclusion, the social background of nationwide fitness and implementation of urban community public service have certain promotion relationship. In order to further improve implementation effect of urban community public sports service, it is necessary to establish sound public service evaluation system, provide corresponding guarantee for community sports activity, boost improvement of physical quality of the public and make corresponding contributions to harmonious and stable development of the society.

\section{Acknowledgments}

This paper is a part of scientific research project of Yunnan Education Department in 2015; project title"Study on Development and Utilization of Baoshan Sports Resources under Background of Public Culture Service System Demonstration Area Construction"; project No.: 2015Y475

\section{References}

[1] Zhang Xinqu, Research on Spatial Arrangement of Nationwide Fitness Path in Hongshan District of Wuhan. Central China Normal University, 2013.

[2] Wang Mingxia, Study on Construction and Practice of Nationwide Fitness Service System Based on Scientific Development Outlook - Case Study of Qingdao Citywide Fitness Status. Shandong Physical Education Institute, 2013.

[3] Dang Linyan, Study on Current Situation of Community Youth Public Sports Activity in Jinshui District of Zhengzhou under Promotion of Nationwide Fitness. Sports Time, 2015(4):43-44.

[4] Yin Dongjun, Research on Endogenous Development of Community Public Sports Service Case Study of Jing'an District, Shanghai. Shanghai Normal University, 2013.

[5] Wu Yuanmeng, Shanghai Normal University of Community Public Sports Service Supply in Dongcheng District of Beijing - Case Study of Community in Beixinqiao Street. Henan University, 2013 